\title{
O PERFIL LIPÍDICO E A SÍNDROME METABÓLICA
}

A doença cardiovascular é reconhecida como a maior causa de morbidade e de mortalidade, especialmente nas últimas décadas, quando tem sido observado um aumento da expectativa de vida da população ${ }^{1,2}$. Procurando reduzir sua incidência, muitos esforços têm sido feitos para se obter um maior conhecimento da sua fisiopatologia e identificar os fatores que condicionam o seu desenvolvimento, de forma a permitir não somente o aumento da longevidade mas também a qualidade de vida das pessoas. Já no início do século passado diversos autores observaram uma associação do risco da doença cardiovascular a alguns fatores como a obesidade, a hipertensão, o diabetes e a dislipidemia, que frequentemente estavam agrupados ${ }^{3,4}$. Posteriormente, novos estudos indicaram que a presença do grupamento destes fatores de risco se relacionavam geralmente à obesidade, especialmente a abdominal ${ }^{3,4}$. Na década de 1950 Vague enfatizou a importância da deposição de gordura a nível abdominal, descrevendo um maior risco de agrupar estes fatores de risco ao perfil antropométrico masculino que apresentava uma maior deposição gordurosa a nível abdominal caracterizando nos indivíduos os perfis de obesidade andróide e ginecóide ${ }^{3}$. 0 impacto destes fatores de risco passaram gradualmente a despertar uma crescente importância por parte de vários autores como causa de mortalidade da população adulta após a quarta década de vida, a ponto de Kaplan et al. descreveram a associação da hipertensão, obesidade, dislipidemia e o diabetes como o "quarteto mortal"3. Entretanto, apesar de reconhecida do ponto de vista epidemiológico, somente em 1988 o conceito de um elo fisiopatológico comum, que segundo a proposta de $G$. Reaven seria o estado de resistência periférica à ação da insulina e a hiperinsulinemia a ela compensatória. Desde então, a resistência à insulina tem sido amplamente reconhecida como o fator fisiopatológico de base para o desenvolvimento de diversas alterações metabólicas que constituem fatores de risco não somente para a doença cardiovascular mas também para o diabetes, a esteatose hepática e as hepatopatias não-alcoólicas 3,4,5,6,7. A presença deste agrupamento de fatores de risco para o diabetes e a doença cardiovascular tem recebido diferentes denominações que a caracterizam como um uma síndrome. Inicialmente designada por Reaven como "Síndrome X" , recebeu outras deteminações como síndrome do Novo Mundo, síndrome da resistência à insulina, síndrome plurimetabólica e finalmente síndrome metabólica, denominação atual e universalmente utilizada ${ }^{3}$. Apesar que, recentemente, a própria existência da síndrome metabólica como uma entidade sindrômica esteja sendo contestada. Considerando que o risco cardiovascular e metabólico estaria condicionado à presença e à intensidade casual de cada um destes fatores, é plenamente comprovado que a resistência à insulina esteja associada a vários fatores de risco e a um processo inflamatório crônico e de hiperoxidação que levaria aos processos de aterosclerose e de lesão celular, especialmente das células beta pancreáticas ${ }^{8,9}$.

Entretanto, o diagnóstico clínico da resistência à insulina que é geralmente inferido em apenas pacientes que apresentam os fatores de risco, especialmente a obesidade, raramente é comprovado na prática clínica rotineira, considerando que as metodologias diagnósticas validadas como as técnicas de "clamp" ou de "modelo mínimo de Bergman", são de execução complexa e elevado custo e as técnicas indiretas como o HOMA, apesar de serem de fácil execução, são mais apropriadas para estudos epidemiológicos, sendo a sua confiabilidade questionada para o uso clínico individual. Dentre os fatores de risco que mais evidências clínicas são descritas destacam-se a hipertensão e a dislipidemia $6,7,10$

A dislipidemia, especialmente a hipercolesterolemia, apresenta uma clara associação com a doença cardiovascular, e vários estudos randomizados realizados demonstram que 0 tratamento com estatinas reduz significantemente o risco de eventos cardiovasculares em pacientes diabéticos ${ }^{11}$. Entretanto, como muito pacientes que apresentaram episódios coronarianos e cérebrovasculares agudos não apresentaram alterações evidentes nos níveis do colesterol ou do LDL-colesterol e que um número expressivo dos pacientes com síndrome metabólica e resistência à insulina apresentaram predominantemente aumento dos níveis de triglicérides e de redução do HDL-colesterol , inúmeros novos estudos estão sendo desenvolvidos no sentido de melhor conhecer a fisiopatologia dos lípides no estado de resistência insulina, de modo a melhor detectar clinicamente o risco destes pacientes ${ }^{12,13}$.

Dentro desta linha de investigação, o estudo "Indicadores do perfil plasmático relacionado à resistência à insulina", de Vasques et al. apresentado nesta edição procura avaliar a habilidade de indicadores do perfil lipídico que permita, por meio de um procedimento de fácil aplicabilidade e de baixo custo, identificar a presença da resistência à insulina em pacientes clinicamente saudáveis. Baseado em seus resultados, os autores concluem que a relação TG/HDL foi o melhor indicador bioquímico do perfil lipídico na avaliação dos níveis mais elevados da resistência a insulina, parecendo ser mais eficiente que a determinação do HDL-colesterol ou hipertrigliceridemia isolados. Entretanto, como sugerem nas suas conclusões, um estudo epidemiológico mais amplo, que utilize maior casuística, e que focalize também alguns extratos específicos da população, são necessários para a sua aplicação. Sugerem ainda que para a utilização clínica deste indicador, o comportamento da relação TG/HDL-C deva ser avaliado em níveis mais altos de TG séricos, para possibilitar a determinação de pontos de corte. Apesar de não haver dúvidas quanto à associação com a dislipidemia e o seu papel no desenvolvimento da aterosclerose e do diabetes, muitas respostas ainda deverão ser obtidas através de estudos adicionais.

\section{Antonio Carlos Lerario}

Professor livre-docente de Endocrinologia da Faculdade de Medicina da Universidade de São Paulo - USP, Diretor da Sociedade Brasileira de Diabetes e médico do Núcleo de Diabetes e Coração do Instituto do Coração - INCOR do Hospital das clínicas da Faculdade de Medicina da Universidade de São Paulo - FMUSP, São Paulo, SP 
Roberto Tadeu Barcellos Betti Doutor em cardiologia pela Faculdade de Medicina da Universidade de São Paulo - FMUSP, Coordenador do Departamento de Diabetes e Doenças Circulatórias da Sociedade Brasileira de Diabetes e médico do Núcleo de Diabetes e Coração do Instituto do Coração - INCOR do Hospital das clínicas da Faculdade de Medicina da Universidade de São Paulo FMUSP, São Paulo, SP

Bernardo Leo Wajchenberg Professor Titular Emérito de Endocrinologia da Faculdade de Medicina da Universidade de São Paulo - USP e médico do Núcleo de Diabetes e Coração do Instituto do Coração - INCOR do Hospital das clínicas da Faculdade de Medicina da Universidade de São Paulo - FMUSP, São Paulo, SP

\section{Correspondência:}

Incor HC-FMUSP

Núcleo de Diabetes e Coração

Av. Dr Enéas Carvalho de Aguiar, nº 44

05403-000 - Cerqueira Cesar - S. Paulo - SP

\section{Referências}

1. Isomaa B, Almgren P Tuomi T, Forsen B, Lahti K, Nissen M, et al. Cardiovascular morbidity and mortality associated with the metabolic syndrome. Diabetes Care. 2001;24:683-9.

2. Kahn R,Buse J, Ferrannini E, Stern M The metabolic Syndrome: time for critical appraisal. Diabetes Care. 2005;28:2289-304.
3. McLaughlin T, Abbasi F, Cheal K, Chu J, Lamendola C, Reaven G. Use of metabolic markers to identify overweight individuals who are insulin resistant. Ann Intern Med. 2003;139:802-9.

4. McLaughlin T, Reaven G, Abbasi F, Lamendola C, Saad M, Waters D, et al. Is there a simple way to identify insulin-resistant individuals at increased risk of cardiovascular disease? Am J Cardiol. 2005;96:399-404.

5. Hannon TS, Bacha F, Lee SJ, Janosky J, Arslanian SA. Use of markers of dyslipidemia to identify overweight youth with insulin resistance. Pediatr Diabetes. 2006; 7:260-6.

6. Summer AE, Cowie CC. Ethnic differences in the ability of triglyceride levels to identify insulin resistance. Atherosclerosis. 2008;196:696-703.

7. Bonora E, Kiechl S, Willeit J, Oberhollenzer F, Egger G, Targher G, et al. Prevalence of insulin resistance in metabolic disorders: the Bruneck Study. Diabetes. 1998; 47:1643-9.

8. Oliveira EP, Lima MD, Souza ML. Metabolic syndrome, its phenotypes, and insulin resistance by HOMA-IR. Arq. Bras Endocrinol Metab. 2007;51:1506-15.

9. Shoelson SE, Lee J, Goldfine AB. Inflammation and insulin resistance. J Clin Invest. 2006;116:1793-801.

10. Semenkovich CF. Insulin resistance and atherosclerosis. J Clin Invest. 2006;116:1813-22.

11. Zavaroni I, Dall'Aglio E, Alpi O, Bruschi F, Bonora E, Pezzarossa A, et al. Evidence for an independent relationship between plasma insulin and concentration of high density lipoprotein cholesterol and triglyceride. Atherosclerosis. 1985;55:259-66.

12. Lakka HM, Laaksonen DE, Lalla TA, Niskanen LK, Kumpusalo E, Tuomilehto J,et al. The metabolic syndrome and total and cardiovascular disease mortality in middle-aged men. JAMA. 2002;288:2709-16.

13. Stern SE, Williams K, Ferrannini E, Defronzo RA, Bogardus C, Stern MP. Identification of individuals with insulin resistance using routine clinical measurements. Diabetes. 2005;54:333-9.

14. Vasques ACJ, Rosado LEFP, Rosado GP, Ribeiro RCL, Franceschini SCC, Geloneze B, Priore SE, Oliveira DR. Rev.Assoc. Med.Bras.2009;55(3): 342-6. 